\title{
MANIFESTACIONES SOBERANAS DE VIDA ¿UN ELEMENTO FUNDAMENTAL DE LA EXISTENCIA O UN MILAGRO?
}

\section{SOVEREIGN MANIFESTATIONS OF LIFE - AN ELEMENTAL PART OF LIFE OR A MIRACLE?}

\author{
Kristian-Alberto Lykke Cobos \\ Universidad de Aarhus (Dinamarca)
}

Recibido: 20-05-2012

Aceptado: 26-06-2012

Resumen: Esta comunicación concluye que a causa de los fenómenos funda $\neg$ mentaleséticoselserhumanoestá entregado mutua-yfundamentalmente. Y que de esto surge una exigencia ética. Las manifestaciones soberanas de vida son suspendidas en la situación de guerra, pero también están bajo presión en nuestra sociedad globalizada, donde constantemente tenemos que reservarnos y protegernos en contra de abusos. Sin embargo, la comunicación también con $\neg$ cluye que la confianza fundamental todavía está en vigor como un elemento fundamental en la existencia y no sólo como un milagro que raramente aparece en la vida del individuo.

La comunicación esta estructurada de la siguiente manera: Primero se explicará el análisis de Løgstrup sobre las manifestaciones soberanas de vida, con la mi $\neg$ rada puesta especialmente en la confianza, así como también en la exigencia ética que acompaña a la existencia. A continuación se demostrará como las manifestaciones soberanas de vida son afectadas en la situación agravada, en la cual en la mayoría de los casos son suspendidas y la vida se convierte en algo insoportable. Después la comunicación implicará la interpretación que Bauman hace del análisis de Løgstrup para demostrar, como la suspensión de confianza no sólo ocurre en la situación agravada, en situación de guerra, sino que también ocurre en nuestro día a día en un mundo globalizado. Y como últi $\neg$ mo se valorará la interpretación de Bauman y se cuestionará.

Palabras-clave: Trust, Ethics, Ethics of War, Sovereign Manifestations of Life, Globalization, War, Peace, Philosophical Anthropology, Zygmunt Bauman, Knud Løgstrup. 


\begin{abstract}
It is important to understand the implications that a critical situation has in a human life. This paper focuses on what happens with the fundamental ethical conditions of a human life in a critical situation, here the situation of war. The paper will defend the following four thesis': Firstly, The ethical demand and the sovereign manifestations of life - that is, the human life demands that we take care of the life of the other and this is the fact from which ethical demand arises is a reality to which we have to react to. Secondly, the sovereign manifestations of life - e.g. the trust, veracity and mercy - are suspended en the critical situation. Thirdly, Zygmunt Bauman underestimates the fundamental character of the sovereign manifestations of life in his interpretation of the analysis that Knud Løgstrup makes on the critical situation. And finally, the fundamental trust still exists in a globalized world and therefore it is not a miracle when it appears.

The paper is structured thusly: first, Løgstrup's analysis of the sovereign manifestations of life is presented which a special focus on the fundamental ethical phenomenon of trust. Secondly, it is demonstrated how the sovereign manifestations of life are affected in the critical situation, and how they are almost always suspended and life is turned into something insufferable. Thirdly, the paper will present Bauman's interpretation of Løgstrup in which Bauman makes clear that he thinks that the sovereign manifestations of life are suspended not only in the critical situation, that of war, but also in our daily life in this globalised world. Finally, this interpretation is evaluated and rejected.
\end{abstract}

Key-words: Trust, Ethics, Ethics of War, Sovereign Manifestations of Life, Globalization, War, Peace, Philosophical Anthropology, Zygmunt Bauman, Knud Løgstrup.

\title{
Introducción:
}

Es importante entender las implicaciones que tiene la situación agravada en la vida humana. Esta comunicación sobre todo enfocará lo que ocurre con las condiciones fundamentales éticas en una situación agravada, una situación de guerra. La comunicación tratará lo que pasa con la confianza, uno de los fenómenos fundamentales. Se argumentará que el ser humano pierde la confianza en la vida en la situación de guerra. Pero también se planteará si la situación de paz se salva de la suspensión de la confianza. O si el ser humano en medio de un mundo globalizado también está afectado por la perdida de confianza al ver las catástrofes humanitarias y económicas.

Esta comunicación defenderá las siguientes cuatro tesis: $1^{\circ}$ La exigencia ética y las manifestaciones soberanas de vida - es decir, que la vida humana exige que salvaguardemos la vida del otro y el hecho de la cual la exigencia surge - es una realidad ante la cual nos debemos definir. $2^{\circ}$ Las manifestaciones soberanas de vida - por ejemplo la confianza, la veracidad y la caridad - se suspenden en la situación agravada. $3^{\circ}$ Zygmunt Bauman subestima la fundamentalidad de las manifestaciones soberanas de vida en la interpretación, 
que él hace, del análisis de Knud Løgstrup sobre la situación agravada. Y por consiguiente: $4^{\circ}$ La confianza fundamental todavía está en vigor en un mundo globalizado y por tanto no es un milagro excepcional cuando aparece.

La comunicación tiene la siguiente estructura: Primero se explicará el análisis de Løgstrup sobre las manifestaciones soberanas de vida, con la mirada puesta especialmente en la confianza, así como también en la exigencia ética que acompaña a la existencia. A continuación se demostrará como las manifestaciones soberanas de vida son afectadas en la situación agravada, en la cual en la mayoría de los casos son suspendidas y la vida se convierte en algo insoportable. Después la comunicación implicará la interpretación que Bauman hace del análisis de Løgstrup para demostrar, como la suspensión de confianza no sólo ocurre en la situación agravada, en situación de guerra, sino que también ocurre en nuestro día a día en un mundo globalizado. Y como último se valorará la interpretación de Bauman y se cuestionará.

La comunicación podrá concluir que: a causa de los fenómenos fundamentales éticos el ser humano está entregado mutua- y fundamentalmente. Y que de esto surge una exigencia ética. Las manifestaciones soberanas de vida son suspendidas en la situación de guerra, pero también están bajo presión en nuestra sociedad globalizada, donde constantemente tenemos que reservarnos y protegernos en contra de abusos. Sin embargo, la comunicación también concluye que la confianza fundamental todavía está en vigor como un elemento fundamental en la existencia y no sólo como un milagro que raramente aparece en la vida del individuo.

\section{La teoría de Løgstrup:}

La primera sección explica el análisis del filósofo danés Knud Løgstrup sobre los fenómenos fundamentales éticos. Los dos términos más importantes que explica esta sección es 'la exigencia ética y 'las manifestaciones soberanas de vida`. De esta manera se motiva la primera tesis: `La exigencia ética y las manifestaciones soberanas de vida son una realidad ante la cual nos debemos definir'.

Desde un análisis fenomenológico Løgstrup toma su punto de partida en las situaciones cotidianas enfocando el encuentro entre seres humanos. En este encuentro identifica varios fenómenos éticos fundamentales, llamadas; las manifestaciones soberanas de vida. La primera es la confianza y esta confianza fundamental es un hecho. El ser humano ya de antemano dispensa confianza al dirigirse al otro, sea expresándose directamente o tan sólo con el lenguaje corporal. El ser humano cuenta con que el otro le responda sinceramente si le pregunta por la hora en la estación de autobuses. Sin duda, no obstante, podemos desconfiar de éste individuo en la parada del autobús. Quizás sea su vestimenta, su aspecto etc. Pero esta desconfianza siempre deriva de otra cosa,

THÉMATA. Revista de Filosofía, $\mathrm{N}^{\circ} 48$ julio-diciembre (2013) pp.: 75-84 doi: 10.12795/themata.2013.i48.06 
deriva del prejuicio que puede ser malo o bueno, pero este no es fundamental. Este se aprende. En cualquier conversación se dispensa confianza; “(...) en el ejercicio al dirigirse a alguien en sí - sin que importe el contenido de la conversación - se entona un tono determinado, en el cual aquél quien habla, por decirlo así, sale de sí mismo para entregarse a la conversación"(Løgstrup 1956:24) ${ }^{1}$. Dicho poéticamente: "El individuo nunca tiene que ver con otra persona sin tener algo de la vida del otro en su mano"(Løgstrup 1956:25-26). La autoentrega ya existe de antemano y esta manifestación de vida es soberana porque no se puede retener, sino que surge espontáneamente. Por eso los seres humanos son interdependientes. Cuando el ser humano está entregado mutuamente surge una relación de poder. El otro está a merced de uno, ya que uno determina si la vida del otro va a realizarse o no(Løgstrup 1956:65). Siguiendo el ejemplo hipotético, el individuo podría mentir y decir que el autobús ya se marchó. Justamente del encuentro surge la exigencia ética. Es una exigencia de amor: has de salvaguardar la vida del otro del mejor modo y por el otro(Løgstrup 1956:59). La exigencia tiene cuatro momentos; silente, radical, unilateral y irrealizable.

Silente. Cuando al ser humano se le pide algo se trata de exigencias explícitas. En cambio, la exigencia ética es implícita y está en un nivel más profundo. "Es parte de la exigencia que el individuo entienda en qué consiste la exigencia, con los conocimientos que pueda tener, con su imaginación y su comprensión"(Løgstrup 1956:32). Uno ha de responder al otro según pueda, lo cual significa, que el otro es limitado o ayudado por el entendimiento de vida y experiencia de individuo. Por eso surgen dos polos entre los cuales cualquier acción se sitúa: consideraciones al otro, con riesgo de indulgencia, y falta de consideraciones al otro, con riesgo de imponerle al otro nuestros propios criterios y formas de pensar, ocasionando agresiones. En ésto consiste la relación de poder. Pero "la responsabilidad por el otro nunca puede consistir en privarle al otro de su propia responsabilidad"(Løgstrup 1956:39). Estámos forzados a mantener el equilibrio entre los dos polos.

Radical. Con otras palabras, la exigencia es incondicional. El primer momento de la radicalidad es que el ser humano no puede evitar la autoentrega, ni dejar de dispensar confianza. El otro se autoentrega y la exigencia ética es clara: uno tiene que accionar de modo altruista por el bienestar del otro. Esto es el segundo momento de la radicalidad; se exige altruismo enteramente.

Unilateral. El fenómeno fundamental de la vida ética es el encuentro: y por ello la dependencia mutua es obvia, pero la exigencia no se trata de hacer lo bueno por la realización común. Únicamente trata de realizar la vida del otro. Si el individuo solamente hiciera lo que le conviene al otro para que su vida en común se realice, entonces estaría reduciendo al otro a un medio de mi propio fin. Sin embargo el otro es un fin en sí mismo. Al individuo se le presenta la

[1] Todas la citaciones están traducidas por mí.

THÉMATA. Revista de Filosofía, $\mathrm{N}^{\circ} 48$ julio-diciembre (2013) pp.: 75-84 doi: 10.12795/themata.2013.i48.06 
exigencia ética del otro sin que pueda reclamar una contrapartida. Pues no se puede reducir al otro a un medio.

Irrealizable. La exigencia ética es irrealizable porque se le demanda al individuo altruismo. Pero el ser humano no puede hacer honor a esta demanda y si lo intentamos nos convertimos en hipócritas. "Visto como exigencia, la exigencia se dirige a la voluntad del ser humano, pero lo paradójico es que la voluntad o el 'yo' no puede ser invocado para cumplir con la exigencia sin que ésta se desplace. (...) El altruismo aparece espontáneamente por sí mismo, o simplemente no aparece"(Fink 2010:15). La exigencia es desplazada en el momento que se hace explicita y el altruismo, por lo tanto, solamente puede presentarse por sí sólo y por culpa del otro.

La culpa o deuda, que Løgstrup trata en relación con la deuda ante la existencia, es lo que Heidegger en Sein und Zeit $\$ 58$ usa para hablar brevemente de la exigencia ética, que en la traducción española se convierte en la exigencia moral(Heidegger 2008:307). La exigencia ética es un existencario. Se podría entender como otra palabra para expresar la idea de Heidegger de Befindlichkiet(encontrarse) combinado con Stimmung(el temple, el estado de animo)(Bauman 2007:119). Lévinas dice que en el encuentro con la cara desnuda del otro surge la exigencia elemental: 'No me mates'(Lévinas 1982:83). Løgstrup se afilia a los teóricos que sitúan lo ético en un nivel pre-ontológico enfocado la idea de la responsabilidad incondicional(Bauman 2007:113).

¿Cómo se distingue la exigencia de exigencias en general? Exigencias están caracterizadas por los momentos contrarios; explicito, condicional, recíproco y realizable. Entre los seres humanos existen una serie de leyes jurídicas, exigencias morales y convenciones sociales. Estas se pueden explicitar. En las leyes de la sociedad encontramos restricciones razonables sobre nuestra conducta. Desde nuestro punto de vista moral, respectivo, encontramos instrucción para cumplir con la exigencia. Y en nuestras convenciones socio-culturales encontramos una codificación de la convivencia. Les exigimos a los demás que cumplan con las normas, y ellos nos lo exigen a nosotros. El otro es reducido a un medio para el bienestar y la protección común. De esta manera vemos la naturaleza condicional, realizable y recíproca de las convenciones. Son el estado de derecho y tratan de garantizar el libre desligue de las manifestaciones soberanas de vida. No es suficiente cumplir con las convenciones para cumplir con la exigencia ética, pero puesto que la exigencia ética en el fondo es irrealizable, las convenciones son relevantes y nos guían para plasmar las palabras y las obras que son necesarias para salvaguardar la vida del otro.

¿Con qué justificación elige el individuo su acción? En sí su contenido es claro, pero no da instrucciones de acción. Desde ella no se puede deducir principios morales. Como salvaguardar al otro depende de la situación, el tiempo, el sitio, el otro y uno mismo. "No tiene otro recurso para entenderlo, sino su propio altruismo y entendimiento de vida"(Løgstrup 1956:69). El ser humano depende

THÉMATA. Revista de Filosofía, $\mathrm{N}^{\circ} 48$ julio-diciembre (2013) pp.: 75-84 doi: 10.12795/themata.2013.i48.06 
enteramente de su experiencia de vida, razón, conocimiento y sobre todo de su imaginación. En la mayoría de los casos el ser humano trata de accionar debidamente y quiere hacerlo con certeza, pero esta voluntad por certeza se convierte en una 'irresponsabilidad absoluta', puesto que "La inteligencia, el conocimiento, y el amor con los cuales se debe accionar en este caso, no son del individuo mismo, sino ahora pertenecen al mensaje radical. El individuo no está involucrado. Sólo existe para realizar, efectuar y usar las indicaciones ya prescritas - sin esfuerzo personal, sin apenas reflexionar y sin tener que tomar una decisión propia"(Løgstrup1956:132). Bauman piensa, que este pasaje demuestra como la incertidumbre es una parte fundamental de la situación ética y no una muestra de imperfección, como clásicamente se ha pensado(Bauman 2007:117). La acción debida nunca se da de antemano, porque la exigencia es silente. Y tampoco sabremos nunca con certeza si cumplimos con ella.

Estos son los momentos y las ideas primordiales de la exigencia ética. De esta manera la primera tesis de la comunicación queda demostrada. La exigencia ética y las manifestaciones soberanas de vida son fundamentales en la existencia y algo ante lo cual nos debemos definir.

\section{La situación agravada - o simplemente la situación de guerra:}

Después de la introducción de la teoría de Løgstrup, la comunicación seguirá con la argumentación de la segunda tesis: 'Las manifestaciones soberanas de vida; la confianza y la veracidad son suspendidas en la situación agravada'.

Empecemos con las definiciones. La situación normal es aquella, cuando a diario vemos personas hundirse junto a una infinidad de injusticias, pero donde hipócritamente podemos imaginarnos que se debe a 'las condiciones` concretas, y por lo tanto no podemos intervenir. La situación agravada, en cambio, es la situación, cuando ya no podemos culpar a 'las condiciones' por las desgracias, y cuando estamos forzados a actuar. Ahora, podemos seguir con el análisis de las consecuencias que tiene la situación agravada para las manifestaciones soberanas de vida.

Løgstrup, como una persona central en la resistencia en Dinamarca en contra de la ocupación Nazi, cuenta como GESTAPO en busca de él se presentaron en la casa de la familia y hablaron con su esposa. Ella obviamente mintió convincentemente y apresuradamente pasaron a la clandestinidad. Lo interesante de este episodio es que las manifestaciones soberanas de vida conscientemente son echadas a un lado por la esposa para salvar a su marido, pero "las manifestaciones soberanas de vida se anticipan a uno, somos presados por ellas. En ello consiste la espontaneidad. También eso lo muestra el episodio. El hombre de la policía secreta del estado intenta aprovecharse de que el habla y la confianza siempre se anticipan a uno - a pesar de que la mujer adivine las 
intenciones y las preguntas y a pesar de su valoración alerta de las consecuencia de cada respuesta"(Løgstrup 1972:17). Las manifestaciones soberanas de vida son suspendidas y temporalmente están en jaque. De un momento a otro la esposa de Løgstrup tuvo que guardarse de decir la verdad. La confianza fundamental y la honestidad natural de la conversación estaban puestas fuera de juego, porque la situación estaba agravada.

En su obra capital Løgstrup usa la resistencia alemana como ejemplo y relata la suerte del resistente Kurt Huber. Éste dijo en su defensa en su juicio que le sentenció a la muerte, lo siguiente: "Como ciudadano alemán, como profesor universitario alemán y como ser humano político aprecio no sólo como mi derecho, sino también como mi deber ético tratar de influenciar el destino de Alemania, poner al descubierto y combatir claros daños." Y reclamaba: "el regreso a principios éticos claros, a el estado de derecho, a la confianza recíproca entre los seres humanos, y esto no es ilegal, sino al contrario el reestablecimiento de la legalidad". "No existe un juicio sobre una comunidad o una nación más terrible que la admisión - la cual estamos todos forzados ha hacer - que nadie se siente seguro ante su vecino, y el padre ya no se siente seguro ante sus hijos"(Løgstrup 1956: 185). El estado de excepción es evidente, los fenómenos ético fundamentales quedan suspendidos, cuando los hijos denuncian a sus padres, porque les han lavado el celebro en Hitler Jugend. Nadie puede sentirse seguro y la vida únicamente puede progresar amputadamente. Las manifestaciones soberanas de vida siempre se anticipan a uno; son fundamentales. Pero esto significa que el individuo constantemente tiene que estar alerta. Ha de reservarse y reprimir su espontaneidad. Así la vida se convierte en algo insoportable y difícil para todo aquél que viva la situación. Cuando el estado de derecho es suspendido, la exigencia ética pierde su protección, pero esta no es suspendida, sino que sigue vigente. Por lo tanto es la responsabilidad de cada uno hacer algo para cambiar la situación. Huber, convencido de esto, concluyó su defensa con las siguientes palabras de Fichte: "Y tienes que reaccionar, como si el destino de las causas alemanas dependieran de ti y de tu acción, y como si la responsabilidad fuera tuya"(Løgstrup 1956: 185). La responsabilidad incondicional es del individuo.

De esta manera la segunda tesis queda demostrada. Las manifestaciones soberanas de vida se suspenden en la situación agravada.

\section{La interpretación de Bauman sobre la teoría de Løgstrup:}

Bauman piensa que la confianza no sólo está suspendida en la situación agravada como excepcionalmente, sino que en cambio la suspensión es ele- 
mento arraigado a la sociedad globalizada. Esto lo argumenta con fenómenos modernos en la relación de pareja y el puesto de trabajo.

Bauman se remite a la descripción de Anthony Giddens de la "pure relationship", la relación de pareja moderna(Giddens1992:58, 137). 'Hasta que la muerte nos separe`se ha convertido en un 'mientras dure`. El individuo es libre para en cualquier momento interrumpir la relación, si siente que esta ya no le aporta nada. Bauman dice que ya no se puede hablar del matrimonio como una 'condición natural'. Aunque todavía sin duda es nuestro ideal, el individuo nunca puede estar seguro de que durará. Por eso se convierte en algo negativo depender del otro. La dependencia cuesta, pues el día que tu pareja te deja, te quedarás herido y sólo.

Tampoco podemos estar seguros de nuestro puesto de trabajo. Las instancias de derecho nacionales no son capaces de combatir la corrupción capitalista, y los seres humanos nada pueden en contra del gran capital: "Robarle a naciones enteras sus recursos se llama 'fomentar el libre comercio'; Robarle a familias y comunidades enteras su fundamento de existencia se llama 'degradación' o simplemente 'racionalización', ninguna de estas acciones están en la lista de acciones punibles y criminales"(Bauman 1999, 119). Ciertamente, Bauman señala que la palabra que usamos todos con gran fervor, la flexibilidad, es problemática.

Por eso Bauman concluye lo siguiente en respecto al análisis de Løgstrup sobre la confianza: "Trust may remain, as Løgstrup suggests, a natural outpouring of the sovereign expression of life-but once emitted it now seeks in vain a place to anchor. Trust is doomed to a life full of frustration (Bauman 2007:125)". La tesis de Bauman es que el ser humano en un mundo globalizado vive bajo una suspensión constante de la confianza fundamental y que la confianza fundamental es un milagro y no una condición de la existencia fundamental. En un mundo globalizado el ser humano ante y sobre todo vive bajo la suspensión de la confianza fundamental, como si estuviera en una situación agravada o de guerra. No podemos confiar ni en el trabajo ni en la pareja.

\section{Problemáticas en la interpretación de Bauman:}

Ahora la comunicación mostrará el problema con la interpretación de Bauman. En la siguiente sección se defenderá la tercera tesis: 'Bauman subestima la fundamentalidad de las manifestaciones soberanas de vida'.

La valoración y la afirmación de Bauman desde mi punto de vista es una subestimación. Bauman señala la importancia del trabajo de Løgstrup y Lévinas; justamente, que los fenómenos éticos son fundamentales, pre-ontológicos. Por consiguiente comete el error de mezclar por un lado la confianza fundamental que no se puede eliminar del programa, cuando sin querer nos autoentregamos y por otro lado la confianza que es convencional. Es una con-

THÉMATA. Revista de Filosofía, $\mathrm{N}^{\circ} 48$ julio-diciembre (2013) pp.: 75-84 doi: 10.12795/themata.2013.i48.06 
fianza que depende de nosotros mismos y es controlada por nuestros prejuicios. La desconfianza se usa como filtro para protegernos. Bauman tiene razón en que la suspensión de la confianza está apegada al ser humano en el mundo globalizado, pero olvida que la confianza fundamental siempre de antemano se anticipa. Esto significa que aunque constantemente vivimos con incertidumbre, no podemos dejar de confiar y entregarnos. Con el creciente grado de incertidumbre y la flexibilidad demandada internalizamos los prejuicios. Aprendemos a reservarnos psíquicamente, pero al tener pareja él o ella se entrega más o menos. Podemos reservarnos en algún tiempo, pero después de unos meses con la misma pareja son confiamos, y la confianza fundamental aparece. Esta confianza puede sufrir una gran ruptura. La ruptura puede ser tan fuerte que la vida se hace insoportable. Pero para eso hay que llegar a un extremo. La mayoría de las personas en un mundo globalizado confrontadas con la miseria del mundo llega a confiar más o menos y se autoentrega. Somos el mundo del otro, y el otro nuestro mundo. Bauman subestima la confianza fundamental, y olvida su fuerza en nuestra mente y conciencia. En términos fenomenológicos mezcla el nivel ontológico con el óntico.

También ha de añadirse, que la situación agravada se diferencia mucho de la situación cotidiana en un mundo globalizado. Las personas en este mundo no sufren traumas de guerra, por ejemplo. La guerra sin duda es más agravada comparando con la situación agravada del día a día en un país occidental. Sin embargo lo importante es ver como la suspensión de la confianza, que normalmente es excepcional, se ha incorporado como algo normal en nuestro día a día. Vemos como ónticamente la confianza fundamental es presionada. No obstante, en el mundo globalizado el ser humano se acostumbra a ver las injusticias y se acostumbra a la suspensión, dejando la confianza desplegarse en las relaciones más cercanas.

\section{Conclusión:}

Así esta comunicación puede concluir lo siguiente: En primer lugar, que el ser humano está entregado mutuamente en confianza y que desde las manifestaciones soberanas de vida surge la exigencia ética de salvaguardar la vida del otro. Esto es una parte fundamental de la existencia y se manifiesta en los fenómenos fundamentales éticos. $1^{\circ}$ La exigencia ética y las manifestaciones soberanas de vida, por lo tanto, son una realidad ante la cual debemos definirnos. Cuando la situación se agrava el ser humano empieza a reservarse y reprimir sus manifestaciones soberanas de vida, lo cual hace la vida insoportable. $2^{\circ}$ La manifestaciones soberanas de vida, la confianza, la veracidad y la caridad, se suspenden en la situación agravada. Bauman radicaliza la suspensión haciéndola vigente constantemente y deduce de allí, que la confianza fundamental es un milagro cuando aparece antes que una parte fundamental de la exis-

THÉMATA. Revista de Filosofía, $\mathrm{N}^{\circ} 48$ julio-diciembre (2013) pp.: 75-84 doi: 10.12795/themata.2013.i48.06 
tencia. Esto se debe a una mezcla entre niveles metafísicos, y a consecuencia $3^{\circ}$ Bauman subestima la fundamentalidad de las manifestaciones soberanas de vida en su interpretación de Løgstrup. Y por lo tanto puedo concluir que $4^{\circ}{ }^{`} L a$ confianza fundamental todavía está en vigor en un mundo globalizado, y por lo tanto no es un milagro excepcional cuando aparece'.

\section{Bibliografia:}

Bauman, Zygmunt.

-2007. Concern for the Other. Indiana: University of Norte Dame Press. (edi. Svend Ander-sen y Kees van Kooten Niekerk)

-1999. Globalisering. De menneskelige konsekvenser. Editorial Hans Reitzels Forlag, Køben-havn. En ingles: 1998. Globalization. The Human Consequences. Cambrigde: Polity Press.

Fink, Hans.

-2007. Livtag med den etiske fordring. Forlaget Klim (Sólo en Danés)

Giddens, Anthony.

-1992. The Transformacion of Intimacy: Sexuality, Love and Erotism in Modern Societies. Cambrigde: Polity Press.

Heidegger, Martin.

-2008(1927). El ser y el tiempo. Fondo de cultura económica, decimocuarta reimpresión.

Lévinas, Emmanuel.

-1982. Etik og uendelighed. Hans Reitzels forlag. En ingles: 1985. Ethics and Infinity: Con-versations with Philippe Nemo. Gazelle Book Services. Løgstrup, Kund Ejler.

-2008 (1956). Den Etiske Fordring(La exigencia ética). Editorial Gyldendal, III versión revisada. En ingles: 1997. The Ethical Demand. Indiana: University of Norte Dame Press.

-1972. Norm og spontanitet. Editoral Gyldendal. 